\title{
Genetic analysis of stilbenoid profiles in grapevine stems reveals a major mQTL hotspot on chromosome 18 associated with disease-resistance motifs
}

Soon L. Teh ${ }^{1,3}$, Bety Rostandy $\mathbb{B}^{1,4}$, Mani Awale ${ }^{2,5}$, James J. Luby ${ }^{1}$, Anne Fennell $\mathbb{B}^{2}$ and Adrian D. Hegeman (1)

\begin{abstract}
Grapevine (Vitis spp.) contains a wealth of phytochemicals that have received considerable attention due to healthpromoting properties and biological activities as phytoalexins. To date, the genetic basis of the quantitative variations for these potentially beneficial compounds has been limited. Here, metabolic quantitative trait locus (mQTL) mapping was conducted using grapevine stems of a segregating $F_{2}$ population. Metabolic profiling of grapevine stems was performed using liquid chromatography-high-resolution mass spectrometry (LC-HRMS), resulting in the detection of 1317 ions/features. In total, 19 of these features matched with literature-reported stilbenoid masses and were genetically mapped using a 1449-SNP linkage map and R/qtl software, resulting in the identification of four mQTLs. Two large-effect mQTLs that corresponded to a stilbenoid dimer and a trimer were mapped on chromosome 18, accounting for phenotypic variances of $29.0 \%$ and $38.4 \%$. Functional annotations of these large-effect mQTLs on the VitisNet network database revealed a major hotspot of disease-resistance motifs on chromosome 18. This 2.8-Mbp region contains 48 genes with R-gene motifs, including variants of TIR, NBS, and LRR, that might potentially confer resistance to powdery mildew, downy mildew, or other pathogens. The locus also encompasses genes associated with flavonoid and biosynthetic pathways that are likely involved in the production of secondary metabolites, including phytoalexins. In addition, haplotype dosage effects of the five mQTLs further characterized the genomic regions for differential production of stilbenoids that can be applied in resistance breeding through manipulation of stilbenoid production in planta.
\end{abstract}

\section{Introduction}

Grapes have been recognized as a rich source of phytochemicals, such as phenolic compounds, which are beneficial to plant and human health. A single glass of red wine that is obtained from the fermented extract of 100 to 140 berries contains up to $500 \mathrm{mg}$ of polyphenolic compounds, depending on varieties and vinification

Correspondence: Adrian D. Hegeman (hegem007@umn.edu)

'Department of Horticultural Science, University of Minnesota, Saint Paul, MN 55108, USA

${ }^{2}$ Agronomy, Horticulture and Plant Science Department, South Dakota State University, Brookings, SD 57007, USA

Full list of author information is available at the end of the article. methods ${ }^{1,2}$. Within the diversity of polyphenolics, stilbenoids represent a relatively restricted group of phenols that is derived from the general phenylpropanoid pathway, and are found in Vitaceae as well as several other plant families ${ }^{3}$. Stilbenoids have received considerable attention due to (1) epidemiological studies attributing moderate consumption of red wine to health benefits, and (2) biological activities as phytoalexins ${ }^{4-10}$.

The production of stilbenoids can be induced by abiotic (e.g., UV irradiation, mechanical injury), as well as biotic (e.g., fungal pathogens) stresses ${ }^{8-12}$. Hypothesized to protect against pathogen infection, stilbenoid production varies across different plant tissues, in terms

\section{(c) The Author(s) 2019}

(c) (i) Open Access This article is licensed under a Creative Commons Attribution 4.0 International License, which permits use, sharing, adaptation, distribution and reproduction cc) in any medium or format, as long as you give appropriate credit to the original author(s) and the source, provide a link to the Creative Commons license, and indicate if changes were made. The images or other third party material in this article are included in the article's Creative Commons license, unless indicated otherwise in a credit line to the material. If material is not included in the article's Creative Commons license and your intended use is not permitted by statutory regulation or exceeds the permitted use, you will need to obtain permission directly from the copyright holder. To view a copy of this license, visit http://creativecommons.org/licenses/by/4.0/. 
of concentrations and types. For instance, resveratrol is induced in grape leaves and berries, but constitutive expression and accumulation of resveratrol and other stilbenoids, which are hypothesized to protect against fungal infection, take place primarily in stems and roots $^{3}$.

Due to these activities associated with stilbenoids, there has been significant interest in developing the means to artificially manipulate stilbenoid production in planta $^{3,13}$. Although breeding objectives in grapevine vary by region and market targets, the overarching goal of many programs is to combine high-quality fruit traits with improved biotic (e.g., diseases and pests) and abiotic resistance (e.g., climate and environmental adaptation).

The availability of myriad genetic resources has enabled routine application of genetic markers for parent and seedling selection, or marker-assisted breeding. In addition to genomic resources, metabolomics is a field that is receiving more attention in the area of crop breeding. Metabolomics is an analytical field that provides a comprehensive investigation of metabolite variations in a biological system ${ }^{14,15}$. Coupled with resources from genomics and transcriptomics, metabolomics is developing as an integrative functional tool for crop breeding ${ }^{16}$.

In a classical QTL-mapping experiment, two parents are crossed so that the measurable trait of interest segregates in the offspring family and can be statistically associated with the underlying genetic markers to explain the genetic basis of variation for the trait ${ }^{17}$. Despite the advancement and higher-throughput of modern analytical tools, crop breeding and genetics have continued to rely on traditional phenotyping data. There have been limited reports of chemical profiles being used as metabolic traits for mapping experiments using known metabolic targets associated with flavor and aroma ${ }^{18-21}$, amino acid metabolism ${ }^{22}$, or fruit color $^{23}$, or an untargeted strategy for associating leaf metabolites with complex traits ${ }^{24}$, or with insect resistance ${ }^{25}$.

The aim of this study is to elucidate the genetic basis of stilbenoid variability in grapevine stem tissue through genetic mapping of putative stilbenoid compounds based on accurate masses. A facile application of liquid chromatography-high-resolution mass spectrometry (LC-HRMS)-based metabolic profiling was carried out to identify metabolic QTLs in a segregating experimental population that targets stilbenoids by using an analysis strategy that has been shown to be broadly effective for this compound class $^{3}$, and by quantifying features with masses consistent with previously characterized Vitis stilbenoids without a priori knowledge of the specific stilbenoids present in this population.

\section{Results}

Profiling of $F_{2}$ mapping family showed metabolic inheritance and segregation

Metabolic profiling of the grapevine stems using LCHRMS yielded 1317 ions (unique retention time $-m / z$ ion pairings) in the $[\mathrm{M}+\mathrm{H}]^{+}$mode. An inheritance pattern of chemical profiles was observed when comparing total ion chromatograms (TICs) of female grandparent $\left(\mathrm{F}_{0}: V\right.$. riparia (USDA PI 588259)), the parent $\left(\mathrm{F}_{1}: V\right.$. hybrid (169-2)), and a representative $F_{2}$ progeny (Fig. 1). In addition, segregation among the $F_{2}$ progeny was exhibited upon examination of stilbenoid feature distributions (Fig. 2). The metabolic segregation of ions provided the foundation for mQTL mapping by treating each ion as a metabolic trait.

\section{Stilbenoid mQTL mapping}

From the list of 1317 ions, these ions were matched with a library of literature-reported stilbenoid masses (Supplementary Table 1). In total, 19 features matched the library and 17 had sufficient data quality to be used subsequently as metabolic "traits" for mQTL mapping. From 19 features, $4 \mathrm{mQTLs}$ were found for 5 metabolic features (Fig. 3; Table 1) using both interval mapping (IM) and composite interval mapping (CIM) analyses. These features belong to different types of stilbene oligomers. An mQTL for feature M229.1423T503 ( $\mathrm{m} / \mathrm{z}:$ 229.1423; retention time: $503 \mathrm{~s}$ ), a monomeric stilbenoid was mapped on chromosome 11 (2.8-3.5 Mbps) with logarithm of the odds (LOD) scores of 12.33 (IM) and 8.96 (CIM) explaining $4.3 \%$ of phenotypic variance. Another monomeric stilbenoid was mapped on chromosome 14 (27.1-27.9 Mbps) with LODs of 3.87 (IM) and 6.96 (CIM), explaining $3.4 \%$ of phenotypic variance. Marker-trait association for stilbenoid dimer (M453.1357T505) and trimer (M681.2169T759) identified the same mQTL on chromosomes 18 (25.0-27.8 Mbps) with LODs of 6.26 and 7.69 (IM analysis), explaining $29.0 \%$ and $38.4 \%$ of phenotypic variances, respectively. In addition, genetic analysis of a tetramer stilbenoid, M907.2767T765 detected an mQTL on chromosome 18 (11.2-11.5 Mbps) with LODs of 3.32 (IM) and 4.70 (CIM) and phenotypic variance of $7.8 \%$ (Fig. 3; Table 1).

\section{Haplotypic dosage effects of mQTLs}

Construction of dosage-dependent haplotypes at each locus enabled the characterization of additive and dominance effects that were associated with differential levels of stilbenoid production. Haplotypes for M229.1423T503 exhibited no statistical difference regardless of allelic dosage. In contrast, haplotypes for the remaining features exhibited dosage-dependent effects. For mQTLs of M229.2006T789 and M907.2767T765, the allelic effects between the homozygotes (i.e., $\mathrm{A}_{1} \mathrm{~A}_{1}$ versus $\mathrm{A}_{2} \mathrm{~A}_{2}$ ) were 


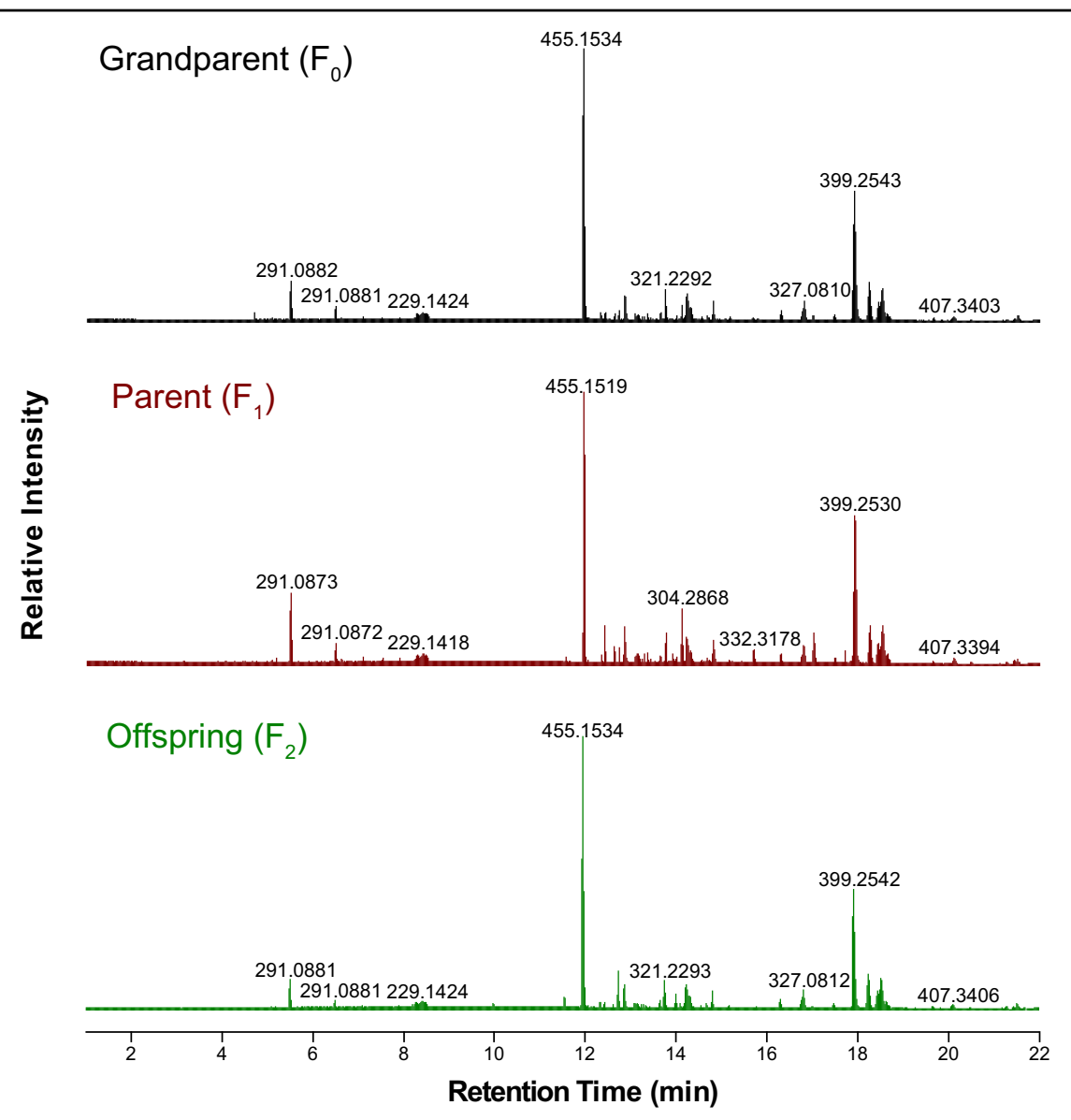

Fig. 1 The total ion chromatograms for grape stem extracts of grandparent $\left(F_{0}:\right.$ V. riparia (USDA PI 588259)), parent $\left(F_{1}: V\right.$. hybrid (16-9-2)), and a representative $F_{2}$ offspring that were analyzed by LC-HRMS

statistically significant and complete dominance was exhibited, where the effect of heterozygote $\left(A_{1} A_{2}\right)$ equaled the effect of homozygous dominant $\left(\mathrm{A}_{2} \mathrm{~A}_{2}\right)$. Meanwhile, haplotypes with partial dominance were observed for mQTL of M681.2169T759 where the effect of $\mathrm{A}_{2} \mathrm{~A}_{2}$ > $A_{1} A_{2}>A_{1} A_{1}$ (Fig. 4).

\section{An mQTL hotspot on chromosome 18 for disease- resistance genes/motifs}

Subsequent to the discovery of mQTLs, the VitisNet database was used to provide functional annotations at the corresponding loci where mQTLs were detected. For the MQTL of feature M229.1423T503, the physical positions span a $0.7-\mathrm{Mbp}$ region on chromosome 11 with 57 annotated genes (54 unique). Three of the genes have roles in disease resistance and plant response to biotic stresses. The mQTL of M229.2006T789 spans a 0.8 -Mbp region on chromosome 14 with 74 functionally annotated genes, of which six genes were involved in plant-pathogen interaction, including diseaseresistance genes. Meanwhile, the mQTL for feature
M907.2767T765 was localized to a $0.3-\mathrm{Mbp}$ region on chromosome 18 with 27 annotated genes. Of these, five genes were annotated as having possible roles in disease resistance and plant biotic stresses (Fig. 3; Table 2; Supplementary File 1).

A major-effect locus explaining $29.0 \%$ and $38.4 \%$ phenotypic variance was co-localized for M453.1357T505 and M681.2169T759, respectively. The mapped physical positions span a $2.7-\mathrm{Mbp}$ on chromosome 18 with 147 functionally annotated genes (Fig. 3). A significant enrichment was identified for the $\mathrm{R}$ proteins from plant-pathogen interaction (vv34627) and the diterpenoid biosynthesis (vv10904) VitisNet pathways, with 13 and 5 genes annotated to the pathway (Table 2; Supplementary File 1). A large number of additional genes not annotated to the pathway but with identified roles in disease resistance were also found in the locus, predominantly Toll/ interleukin-1 receptor (TIR)-nucleotide-binding site (NBS)-leucine-rich repeat (LRR) (Supplementary File 1). In addition, 15 genes were annotated as being involved in flavonoid biosynthetic pathways, as were six genes in 


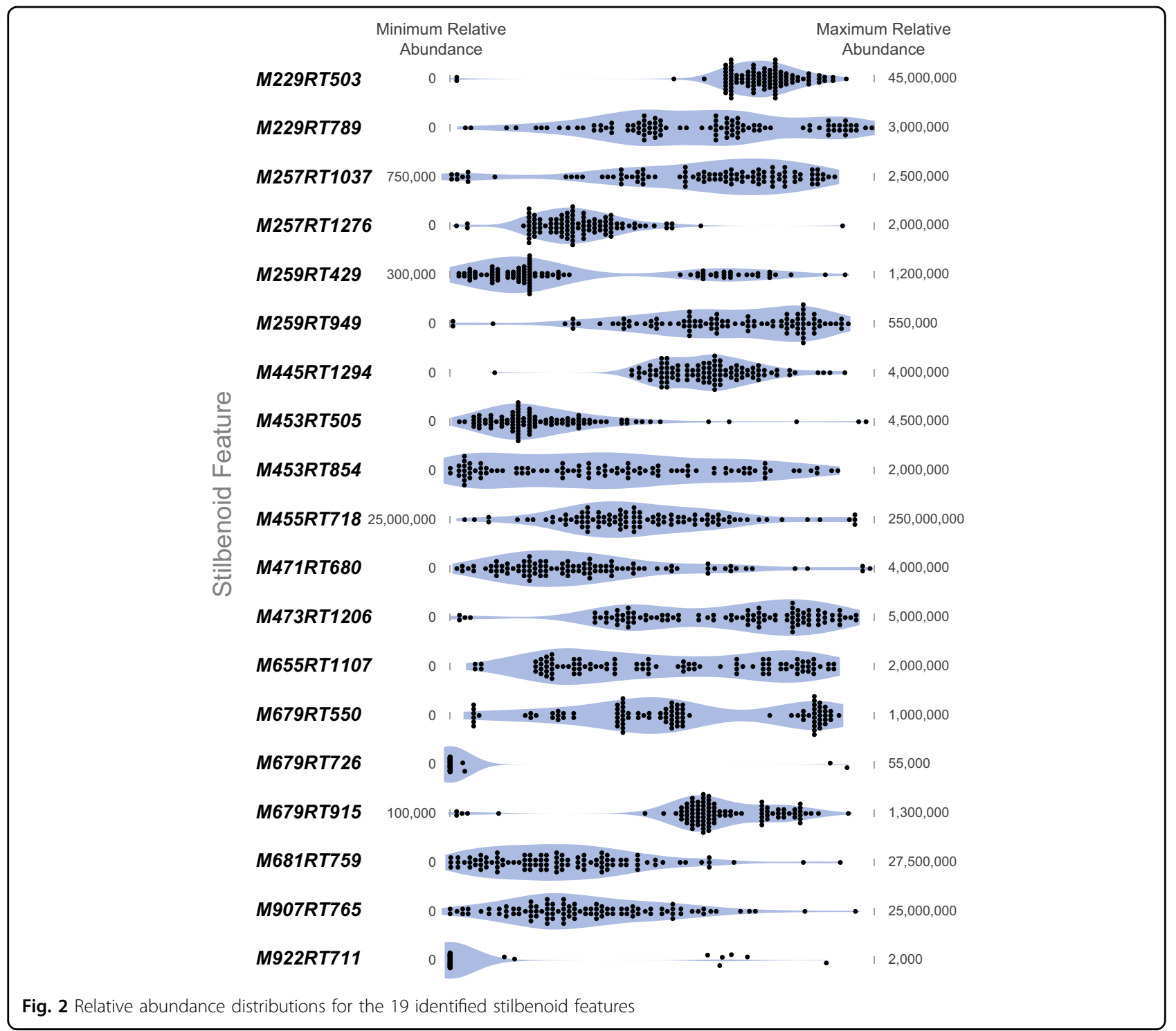

terpenoid biosynthesis with putative function in hydroxylation.

\section{Discussion}

Mapping loci using variation in metabolite levels of the progeny

Genes that are involved in a biosynthesis pathway have been identified either through forward genetics (i.e., screening the phenotype to identify gene(s) responsible for a trait of interest) or reverse genetics (i.e., evaluating the phenotype subsequent to disruption of gene function). In traditional crop breeding, the former case has been routinely applied, resulting in QTL discovery, fine mapping, candidate gene cloning, and finally, functional identification. Despite the eventual identification of genes, it remains unclear which of the genes account for the variation in metabolite levels across tissue types and genotypes. Alternatively, genetic mapping of metabolites provides a solution to elucidate putative genes that underlie metabolic variations.

In this study, an integrated approach in an $\mathrm{F}_{2}$ family of 101 offspring linking a genetic map of 1449 SNPs and a metabolomics data set of 1317 metabolic features that was coupled with a library of stilbenoid masses resulted in the discovery of five mQTLs. In addition, the use of a highresolution mass spectrometer provided accurate mass detection of ions from a complex chemical extract, thereby enabling high-throughput analysis of the mapping family. Without a priori knowledge of compound identity, metabolic profiling of an experimental population can provide thousands of metabolic features to be evaluated in mQTL mapping. Similar to the conventional marker-trait association where marker information is derived from a genetic linkage map while trait information is acquired 


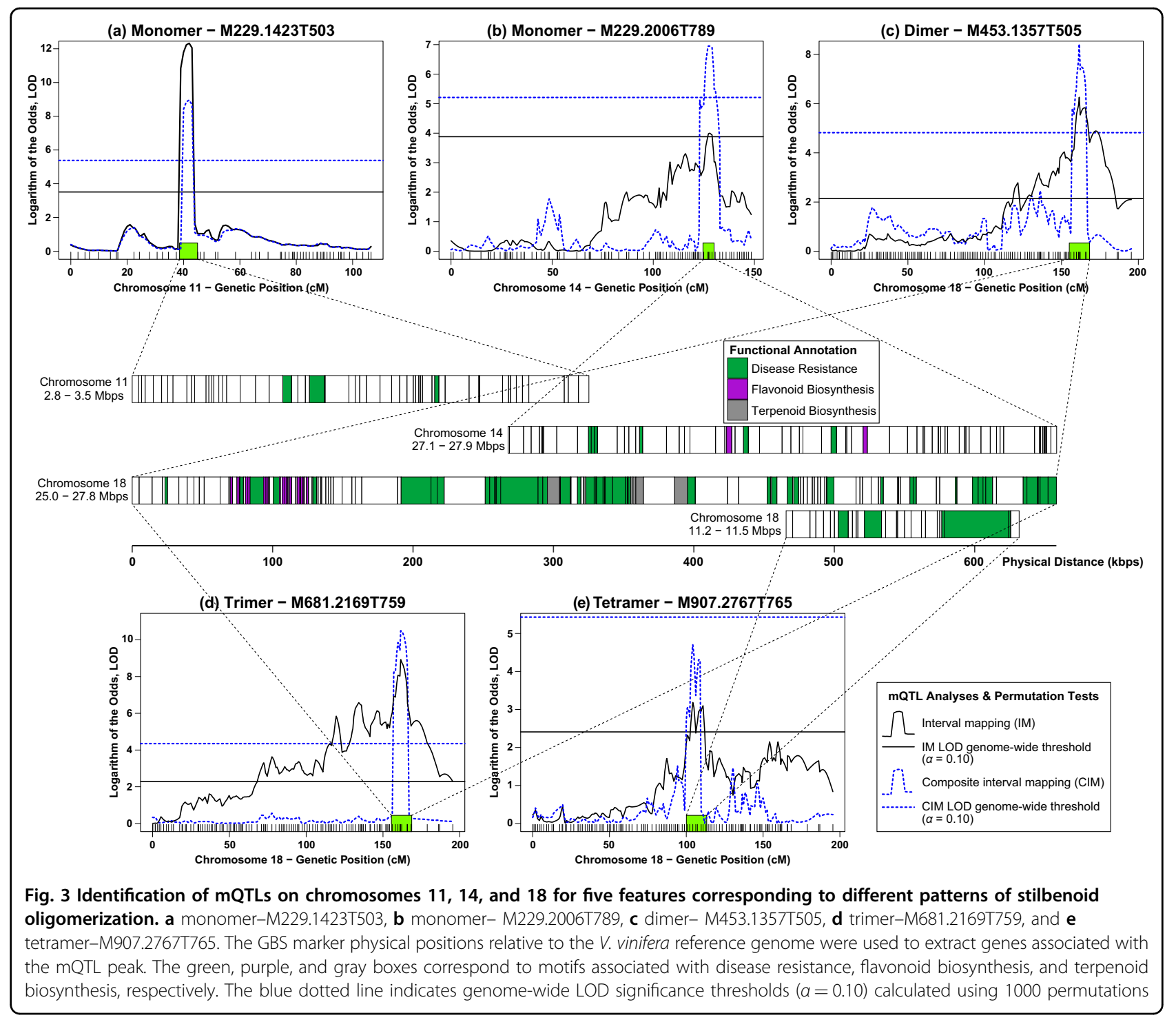

from a variation for phenotype of the trait, a metabolic feature can be treated as a "trait" to assess marker-"trait" (feature) association ${ }^{22,24}$. Significant variation in ion abundance/intensity is apparent across the 19 features (Fig. 2). Since the observed distributions of features are multimodal for segregating traits, and can often be confused with skewed single"-modal distributions, we opted to perform the QTL analysis without transformation. Although not required for our data set, $\log _{2}$ and $\log _{10}$ transformations can be performed to normalize the distribution for other analyses, as needed.

\section{Matching features with a library of stilbenoid masses prior to $\mathrm{mQTL}$ mapping}

From the list of 1317 metabolic features, the vast majority of features did not match with a library of stilbenoid masses that have been reported in the literature
(Supplementary Table 1). This guided approach enabled a targeted list of 19 features to be examined for mQTL mapping. Subsequent $\mathrm{mQTL}$ analysis (with genome-wide threshold of $\alpha=0.10$ ) identified four mQTLs. Despite the suitability of this method, there are several reasons why the number of mQTLs may have been underestimated (i.e., false negatives). First, complex genetic regulation of metabolites in a pathway could hamper the detection of mQTLs. The production of one metabolite is likely controlled by several small-effect loci, which may not be detected during the mapping analysis. In addition, the complex genetic backgrounds of an already highly heterozygous crop may help explain the presence of smalleffect loci, rendering a lower-resolution $\mathrm{MQTL}$ mapping. The ancestry of the $F_{2}$ mapping family used for this experiment is comprised of at least six Vitis species, including $V$. riparia, $V$. vinifera, $V$. rupestris, $V$. labrusca, 


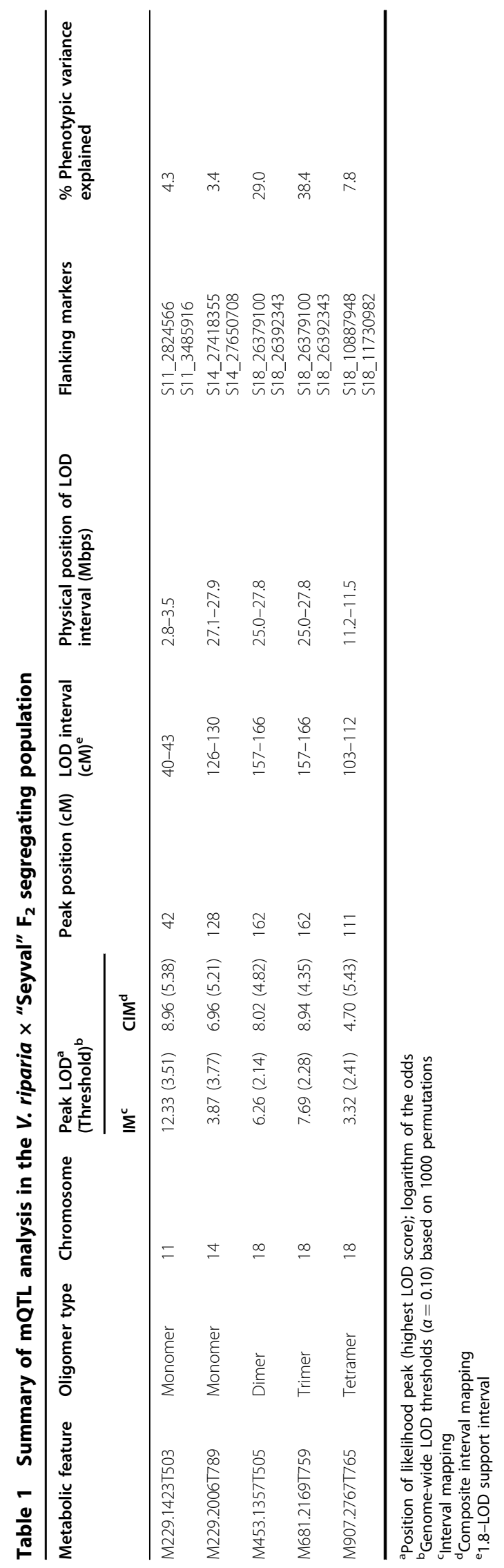

$V$. aestivalis, and $V$. berlandieri. Relevant marker loci of the highly interspecific hybrid progeny can be lost due to anchoring on the $V$. vinifera reference genome during the GBS process. Finally, the ion signal of metabolic features could be above the noise threshold for some offspring, but within the noise threshold for other offspring, likely resulting in no $\mathrm{mQTL}$ detection.

\section{mQTLs on chromosome 18 are coincident with disease- resistance genes/motifs}

In a $2.7-\mathrm{Mbp}$ region on chromosome 18 , a hotspot of disease-resistance genes was jointly detected for features M453.1357T505 and M681.2169T759 (Fig. 3; Table 1). The lack of genetic resolution is likely due to the reduced recombination events in this relatively small $F_{2}$ family. Although the original $F_{2}$ family is comprised 424 progeny, dormant cane sections were acquired from only a subset of this family (i.e., $N=101)^{26}$. A comprehensive analysis using the entire family would likely resolve the mQTLs detected for features M453.1357T505 and M681.2169T759.

From the network database, a third of known genes (Chr. 18: 25.0-27.8 Mbps) were saturated with diseaseresistance motifs, such as TIR-NBS-LRR, TIR-NBS, LRR family protein, and $R$ protein (Table 2 ; Supplementary File $1)$. They were annotated as " $R$ proteins from plant-pathogen interaction" and fell into categories of "biotic stress response", "response to stimuli", "plant-pathogen interaction", as well as "transposable elements". Along with the statistical reports of $29.0 \%$ and $38.4 \%$ phenotypic variance, this genomic region is a major-effect locus for disease resistance, indicating a major potential hotspot for the biosynthesis of defense metabolites.

The detection of a large-effect disease-resistance hotspot coincident with mQTLs for stilbenoid dimers and trimmers, but not for monomers, is consistent with previous findings that the type, rather than the amount of stilbenoids are more important in grapevine resistance to pathogens ${ }^{3,9,10,27,28}$. Though previously touted as an important phytoalexin in plant defense, resveratrol (i.e., a stilbenoid monomer) has been reported to be less fungitoxic than stilbenoid dimers and trimers, such as viniferins $^{9,10}$.

In a recent QTL mapping of downy mildew and stilbenoid induction, Vezzulli et al. reported a list of candidate genes underlying QTLs for downy mildew severity and incidence, also mapped on chromosome $18^{29}$. These candidate genes overlap with our major hotspot of disease-resistance motifs on chromosome 18. In addition in Vezzulli et al.'s findings, most polyphenol-related QTLs were mapped on chromosome 16, while only cis- and trans-piceid, astringin, isorhapontin, $c i s-\varepsilon$-viniferin, and the sum of monomeric stilbenoid abundance were 


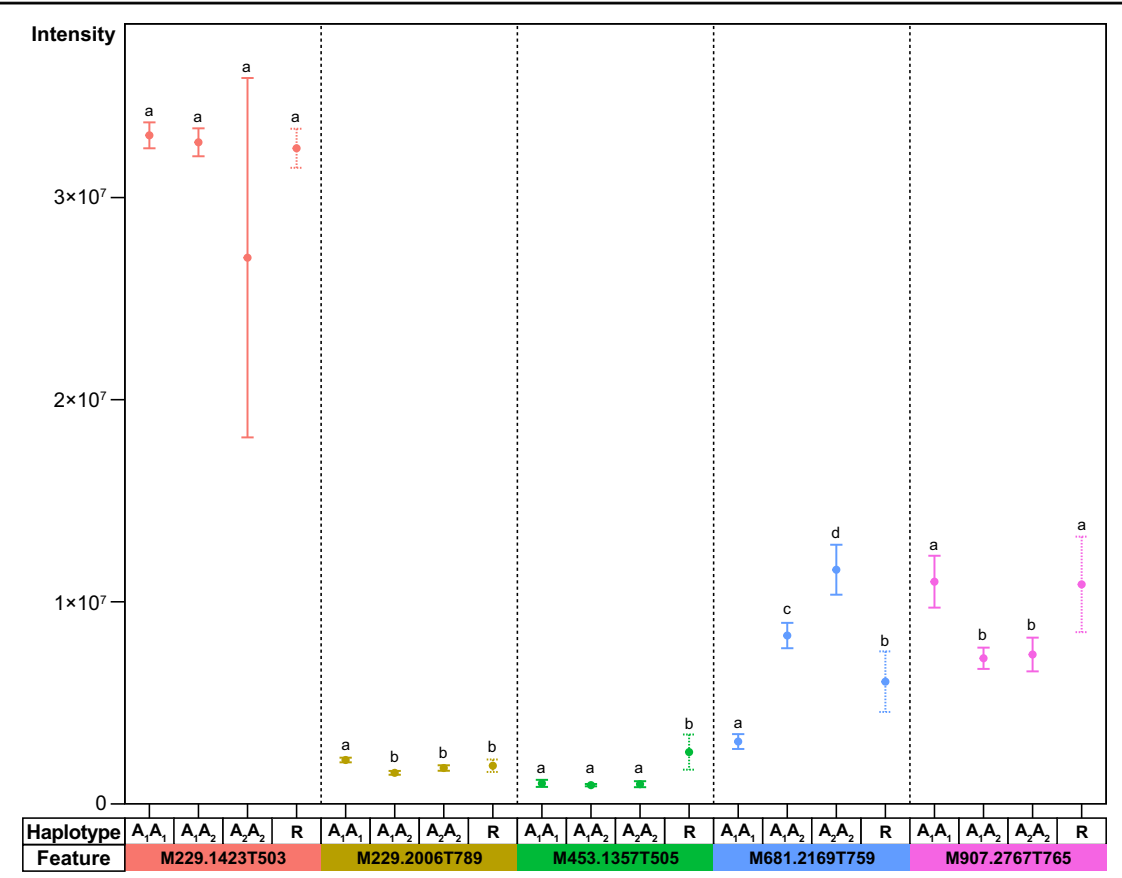

Fig. 4 Haplotypic dosage effects of mQTLs. Haplotypes were denoted in a dosage-dependent manner- $A_{1} A_{1}, A_{1} A_{2}$, and $A_{2} A_{2}$. Recombinant haplotype was designated as "R". Letter assignment indicates statistical significance based on Tukey's significant difference test $(p<0.05)$

mapped on chromosome 18. Stilbenoid mQTLs identified in this study were found on chromosome 18, overlapping the down mildew resistance region described by Vezzulli et al. ${ }^{29}$.

Coincidentally, the identification of a diseaseresistance hotspot on chromosome 18 co-localizes with previous QTL reports for downy mildew and powdery mildew resistance in grapevine. Di Gaspero et al. mapped the Rpv3 (resistance to Plasmopara viticola or downy mildew) locus on chromosome 18 and reported haplotypic structure at six microsatellite loci spanning a $1.2-\mathrm{Mbp}$ region from 24.8 to $26.0 \mathrm{Mbp}$ on chromosome 18, a region overlapping with our reported mQTLs ${ }^{30}$. The Rpv3 locus was also linked with stilbenoid induction in a recent interspecific grapevine population mapping study conducted by Vezzulli et al. ${ }^{29}$. The resistance locus was inherited from "Seyval", which is a grandparent in our $F_{2}$ mapping family. Another report on grapevine downy mildew resistance also detected a major locus on chromosome 18, being strongly associated with GF18-06 and GF18-08 markers that were mapped on the 25.9-26.9-Mbp region ${ }^{31}$. The resistant parent of the experimental population was GF. GA-47-42, which was a cross between "Bacchus" and "Seyval" 31 . Meanwhile, in grapevine powdery mildewresistance mapping, the Run2 (resistance to Uncinula necator) was mapped on chromosome 18 for four traits -leaf, cane, rachis, and fruit ${ }^{32}$. The locus is closely linked to the VMC7f2 marker, anchored at a physical position of $26.9 \mathrm{Mbp}^{32}$, which is located within the 25.0-27.8-Mbp region of disease-resistance motifs.

Taken together, the co-localization of our mQTLs on chromosome 18 with a major disease-resistance hotspot, and three QTL findings on grapevine downy and powdery mildews implied further support that the region is likely associated with downy and powdery mildew resistance. In the case of downy mildew resistance, the resistance donor parent or progenitor in the experimental crosses is "Seyval", the same $F_{0}$ grandparent in our experimental $F_{2}$ segregating family ${ }^{30,31}$. Given the preliminary observations of our analysis, further experiments need to be carried out to characterize the roles of this region in conferring resistance.

In addition to disease-resistance motifs, genes of two other annotated metabolic pathways were quite ubiquitous in this region (Chr. 18: 25.0-27.8 Mbps). Genes involved in flavonoid and terpenoid biosynthetic pathways (Fig. 3), that are involved in the production of various secondary metabolites, including those involved in plant defense response, were associated with the $\operatorname{loc}^{33,34}$.

mQTLs on chromosomes 11,14 , and 18 associated mainly with primary metabolism

With the exclusion of a handful of disease-resistance genes, the genomic regions for mQTLs associated with features M229.1423T503, M229.2006T789, and M907.2767T765 appear to be populated with a suite of genes linked with primary metabolism that are not 
obviously connected to stilbenoid metabolism. In combination with the observation of disease-resistance motifs highlighted in the aforementioned mQTL hotspot, it is likely that these regions may also be associated with plant-pathogen interaction. Secondary metabolism is of great interest in plant-pathogen interaction because phytopathogen infection induces a plant's defense program. However, less is known about the effects of pathogenic attack on primary metabolism. This is especially important because most attacks (e.g., parasitic relationship) result in yield losses without killing the crops. In particular, various aspects of photosynthesis, assimilate partitioning, and source-sink relationship are downstream physiological changes of the infected tissues that need to be investigated to understand the mechanisms and consequences of a plant-pathogen interaction ${ }^{35-37}$.

Based on the network analysis, some of the functional annotations in these genomic windows include the signaling pathway, macromolecule transport, transcription regulation, ubiquitin-mediated proteolysis, nucleic acid metabolism, carbohydrate metabolism, and glycosyl transference (Supplementary File 1 ) $^{38}$. In addition to genes related to primary metabolism, the annotation indicates a peroxidase gene that appears to be involved in the metabolism of phenylalanine, a precursor to various biosynthetic pathways, such as phenylpropanoid, flavonoid, and stilbenoid pathways. Similar peroxidases have been implicated in stilbenoid oligomerization and so may be directly involved in the coupling of higher-order stilbenoids $^{13}$. Taken together, it is likely that these three mQTL regions may be linked with plant-pathogen interaction, both in primary and secondary metabolism.

\section{Conclusion}

In this study, we demonstrated the utility of combining analytical tools (i.e., metabolic profiling) and large genomic data sets to characterize the genetic basis of metabolites. Despite the absence of compound identity and structures, the use of high-resolution mass spectrometer provided detection of various ions that can be treated as metabolic "traits" and coupled with genetic maps for mQTL mapping. Understanding the genetic controls of potentially bioactive compounds (e.g., stilbenoids) can assist breeders and viticulturists to select genotypes (e.g., seedlings, parents, and existing cultivars) with increased levels of these biomarkers through marker-assisted breeding.

\section{Materials and methods}

\section{Sampling of plant materials}

A segregating $F_{2}$ mapping family was derived from selfpollination of a single hermaphrodite $F_{1}$ individual (16-92 ), which was a hybrid from the initial $\mathrm{F}_{0}$ cross of $V$. riparia 37 (USDA PI 588259) × "Seyval” (Seyve-Villard 5-
276), as previously described by Fennell et al. ${ }^{39}$. The experimental family was grown and maintained in the field in Brookings, SD. The original $\mathrm{F}_{2}$ mapping family consists of 424 offspring that has been mapped for QTLs of enological and environmental adaptation traits ${ }^{26,40}$. In this study, a subset $(N=101)$ from the pilot-mapping family was used for metabolic profiling and mQTL mapping. Two to four cuttings of approximately three to five inches of dormant woody stems from each of 101 progeny were collected between February 26, 2014 and March 12, 2014. Samples were wrapped in aluminum foil to prevent moisture loss, shipped on dry ice to Minnesota, and stored at $-80^{\circ} \mathrm{C}$ until extraction and analysis.

\section{Sample preparation and extraction}

Woody stems were lyophilized for 24 to $36 \mathrm{~h}$ until all moisture was removed. For each offspring, the dried stems were ground together with a conventional coffee grinder (Kuissential ${ }^{\mathrm{TM}}$ Ceramic Burr Coffee Grinder). Inbetween samples, the grinder was cleaned and air-dried. The ensuing steps, including chemical extraction, sonication, centrifugation, evaporation, and adjusted reconstitution, were performed based on a facile extraction method that was described by Pawlus et al. ${ }^{27}$.

\section{Metabolic profiling with LC-HRMS}

An aliquot $(1 \mu \mathrm{L})$ of each reconstituted extract $(0.2 \mu \mathrm{g} / \mu \mathrm{L})$ was injected and analyzed on an UltiMate 3000 UHPLC coupled to a Q Exactive Hybrid Quadruple-Orbitrap mass spectrometer (Thermo Fisher Scientific, USA). Samples were chromatographically resolved at a flow rate of $0.45 \mathrm{~mL} / \mathrm{min}$ on a $\mathrm{C}_{18}$-reverse-phase column (HSS T3, $2.1 \mathrm{~mm}$ i.d. $\times 100 \mathrm{~mm}, 1.8-\mu \mathrm{m}$ particle size; Waters, Milford, MA) by mixing mobile-phase solvent $A$ (water with $0.1 \%$ formic acid) and solvent B (acetonitrile with $0.1 \%$ formic acid) to generate the following gradient: 0 to $1 \mathrm{~min}$, $2 \% \mathrm{~B} ; 1$ to $10 \mathrm{~min}, 2$ to $30 \% \mathrm{~B} ; 10$ to $12 \mathrm{~min}, 30$ to $50 \% \mathrm{~B}$; 12 to $20 \mathrm{~min}, 50$ to $75 \% \mathrm{~B} ; 20$ to $22 \mathrm{~min}, 75$ to $98 \% \mathrm{~B} ; 22$ to $23 \mathrm{~min}, 98$ to $2 \% \mathrm{~B} ; 23$ to $27 \mathrm{~min}, 2 \% \mathrm{~B}$. The mass spectrometer was operated in the positive/negative switching ionization mode over a scan range of $150-2000 \mathrm{~m} / z$. The presence of monomers, dimers, trimers, and tetramers was monitored using the $[\mathrm{M}+\mathrm{H}]^{+} m / z$ values of 229 , 445,681 , and 907 , respectively. Ions yielded in the $[\mathrm{M}-\mathrm{H}]^{-}$mode were not used for mQTL mapping due to ionization issues. UV/visible absorbance data were simultaneously collected using the UltiMate 3000 UHPLC diode array detector throughout each separation; spectra with $\lambda_{\max }$ values of $280 \mathrm{~nm}$ and $306 \mathrm{~nm}$ were consistent with eluting stilbenoids.

\section{Data pre-processing}

Metabolomics data from LC-HRMS were processed in XCMS for peak alignment with parameter settings 


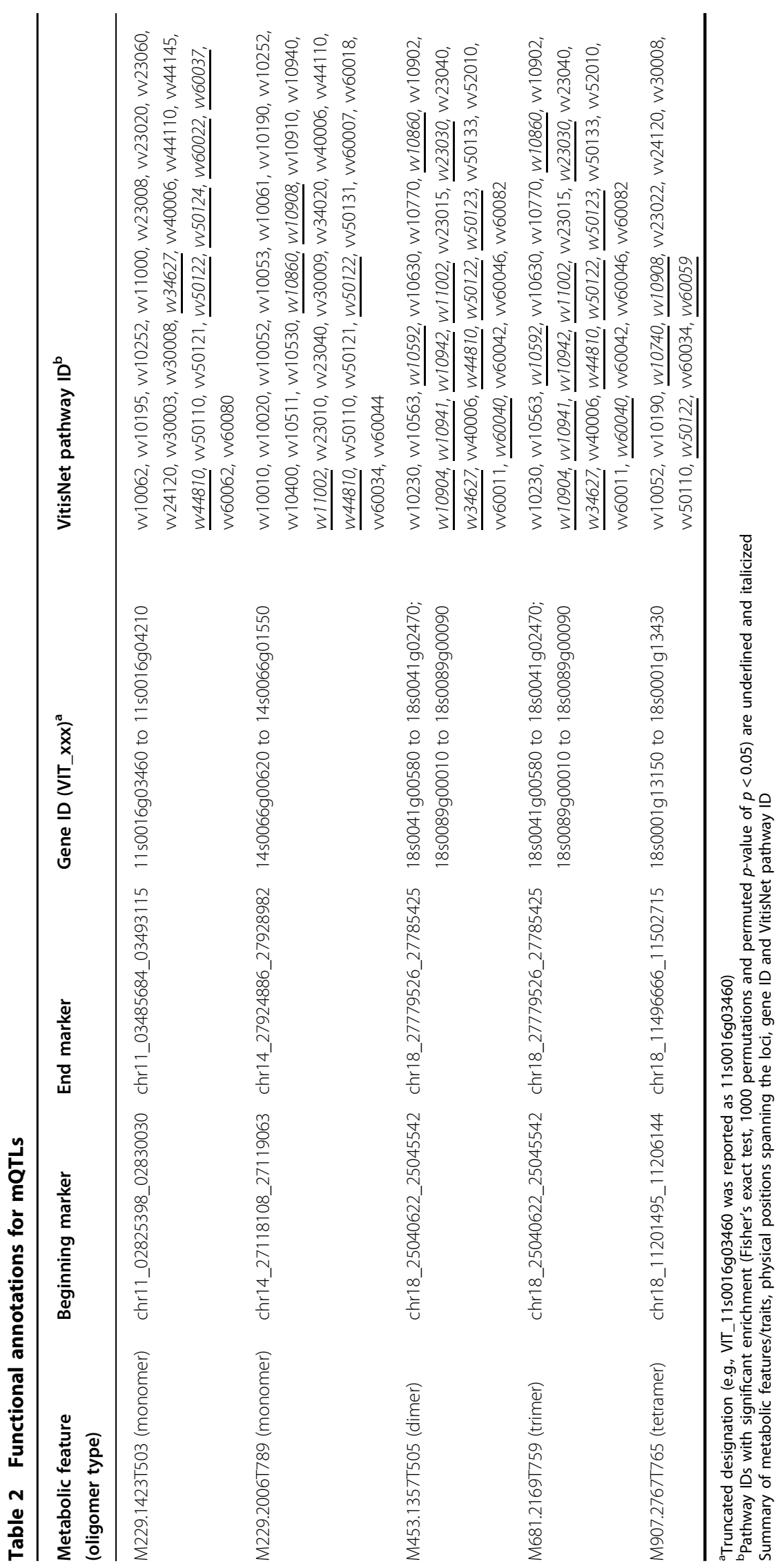


optimized for the analysis (method $=$ centWave; peakwidth $=5,20$; snthresh $=10 ;$ ppm $=3.0 ;$ mzdiff $=0.01)^{41}$. The aligned peaks were then subjected to grouping, retention time correction, and regrouping with optimized parameters $(\mathrm{bw}=2$; mzwid $=0.015$; minfrac $=0.1)$. Following feature grouping and correction, peak filling was performed using chromatography method in XCMS, allowing integration of the area under the curve of samples that might have been missed during the group step.

However, XCMS peak picking software might still miss the reporting of features in samples with lower abundance or absence of metabolite ions, resulting in zero entries in the generated peak table. These zero entries are considered missing values. In this experiment, imputation of missing values was not performed, but features with $>10 \%$ missing values in the offspring samples were not considered for subsequent QTL analysis to avoid potential erroneous QTL detection. Of the 19 stilbenoid features, 14 contain no zero-intensity values, 1 (M453RT854) contains a single zero-intensity value, 2 (M681RT759 \& M907RT765) contain 2 zero-intensity values, and 2 (M922T711 and M679T726) were ultimately excluded with 93 zero-intensity values, and 97 zero-intensity values respectively.

\section{Linking metabolic features with a library of stilbenoid masses}

A list of accurate masses corresponding to 54 structurally characterized stilbenoids was compiled from the literature (Supplementary Table 1). Using this list, 19 features, out of the 1317 extracted using XCMS, were found to match stilbenoid masses from this list. All 19 features were confirmed manually by examining extracted ion chromatograms (EICs) generated from the raw data files using Xcalibur 4.0 data visualization software for each corresponding accurate mass value.

\section{Stilbenoid metabolic quantitative trait locus (mQTL) mapping}

The intensities for each feature (with a mass matching a stilbenoid and a unique retention time) were compiled for each individual for subsequent mQTL mapping; each individual stilbenoid metabolic feature (unique mass/ retention time pair) was treated as a metabolic "trait" for the analysis.

A previously described genotype-by-sequencing singlenucleotide polymorphism (SNP) map with 1449 markers over 19 chromosomes was used for QTL analysis of 17 (2 were excluded with $>10 \%$ missing values) of the 19 identified stilbenoid features ${ }^{26}$. As described by Yang et al., the $\mathrm{F}_{2}$ genetic map is comprised $<$ hkxhk $>$ markers with linkages estimated using cross-pollination cross type ${ }^{26}$. QTLs with a recessive allele that cannot be detected in F1 may be detected in F2 because of the change in segregation type from $<\operatorname{lmxll}>$ or $<$ nnxnp $>$ (2 genotypic classes) to $<$ hkxhk $>$ (three genotypic classes). Targeted mQTL detection was conducted on R/qtl software (version 3.5.3) using interval mapping (IM) and composite interval mapping $(\mathrm{CIM})^{42}$. Interval mapping was performed using scanone function (R/qtl) with expectation-maximization (EM) algorithm. Composite interval mapping was conducted using $\operatorname{cim}()$ function with the Kosambi map function. The minimum logarithm of odds (LOD) score for mQTL detection was determined by genome-wide LOD significance thresholds $(\alpha=0.10)$ calculated using 1000 permutations.

\section{Haplotype construction and analysis of mQTLs}

A haplotype is defined by a combination or a group of alleles that tend to be inherited together. Haplotype was constructed by assigning alleles to offspring in a family based on observed marker alleles that had already been assigned during QTL analysis. At each mQTL, functional SNP haplotype spanning the locus was constructed for the progeny based on differential intensities of the feature. Given that $<\mathrm{hk} \times \mathrm{hk}>$ marker type was used in the SNP map, mQTL haplotyping was reported as haplotypic dosage effects (i.e., $A_{1} A_{1}, A_{1} A_{2}$, and $A_{2} A_{2}$ ). A recombinant haplotype was designated where there was a recombination at the $\mathrm{mQTL}$ region. Analysis of variance (ANOVA) was performed to determine if there was a statistical difference owing to these haplotype dosage effects. A post hoc Tukey's analysis was used to identify significant difference $(p<0.05)$.

\section{Functional annotation of $\mathrm{mQTLs}$}

To gain more insights into the $\mathrm{mQTL}$ regions, the physical positions of the markers defining the mQTLs were used to identify genes associated with the loci using the PN40024 $12 \times . v 2$, V2 annotation ${ }^{38,43}$. Gene functional annotation and VitisNet pathways were used to explore genes underlying the loci of interest. Genes associated with the QTL were tested for enrichment in VitisNet Pathways, using a 1000 permutations, a Fisher's exact test of $p<0.05$ and a permuted $p$-value of $p<0.05^{38,44}$.

\section{Acknowledgements}

Funding for this research was provided by the USDA-NIFA Specialty Crops Research Initiative (Award Nos 2011-51181-30635 and 2011-51181-30850) through the VitisGen project and the Northern Grapes Project, as well as by the Minnesota Agricultural Experiment Station and the South Dakota Agricultural Experiment Station through the Hatch projects. Funding was also provided by the National Science Foundation IOS (Award No. 1238812).

\section{Author details}

${ }^{1}$ Department of Horticultural Science, University of Minnesota, Saint Paul, MN 55108, USA. ${ }^{2}$ Agronomy, Horticulture and Plant Science Department, South Dakota State University, Brookings, SD 57007, USA. ${ }^{3}$ Present address: Tree Fruit Research and Extension Center, Department of Horticulture, Washington State University, Wenatchee, WA 98801, USA. ${ }^{4}$ Present address: Department of Mathematics and Statistics, University of North Carolina, Greensboro, NC 27412, 
USA. ${ }^{5}$ Present address: Grape and Wine Institute, University of Missouri, Columbia, MO 65211, USA

\section{Authors' contributions}

S.L.T., J.J.L., A.F. and A.D.H. designed the experiment. S.L.T., B.R. and M.A. performed the experiment. S.L.T., B.R., M.A. and A.F. conducted the statistical analyses. S.L.T. wrote the paper and all authors contributed to the final draft.

\section{Conflict of interest}

The authors declare that they have no conflict of interest.

Supplementary Information accompanies this paper at (https://doi.org/ 10.1038/s41438-019-0203-x).

Received: 16 April 2019 Revised: 24 September 2019 Accepted: 1 October 2019

Published online: 08 November 2019

\section{References}

1. Lopez, M., Martınez, F., Del Valle, C., Orte, C. \& Miro, M. Analysis of phenolic constituents of biological interest in red wines by high-performance liquid chromatography. J. Chromatogr. A 922, 359-363 (2001).

2. Stervbo, U., Vang, O. \& Bonnesen, C. A review of the content of the putative chemopreventive phytoalexin resveratrol in red wine. Food Chem. 101 449-457 (2007).

3. Pawlus, A. D., Waffo-Téguo, P., Shaver, J. \& Mérillon, J. M. Stilbenoid chemistry from wine and the genus Vitis, a review. J. Int. des. Sci. de. la Vigne et. du Vin. 46, 57-111 (2012).

4. Adrian, M., Jeandet, P., Veneau, J., Weston, L. A. \& Bessis, R. Biological activity of resveratrol, a stilbenic compound from grapevines, against Botrytis cinerea, the causal agent for gray mold. J. Chem. Ecol. 23, 1689-1702 (1997).

5. Anstey, K. J., Mack, H. A. \& Cherbuin, N. Alcohol consumption as a risk factor for dementia and cognitive decline: meta-analysis of prospective studies. Am. J. Geriatr. Psychiatry 17, 542-555 (2009).

6. Chao, C., Slezak, J. M., Caan, B. J. \& Quinn, V. P. Alcoholic beverage intake and risk of lung cancer: the California Men's Health Study. Cancer Epidemiol. Prev. Biomar. 17, 2692-2699 (2008).

7. Wang, J. et al. Moderate consumption of Cabernet Sauvignon attenuates $A \beta$ neuropathology in a mouse model of Alzheimer's disease. FASEB J. 20, 2313-2320 (2006)

8. Mattivi, F. et al. Profiling of resveratrol oligomers, important stress metabolites, accumulating in the leaves of hybrid Vitis vinifera (Merzling $\times$ Teroldego) genotypes infected with Plasmopara viticola. J. Agric. food Chem. 59, 5364-5375 (2011).

9. Pezet, R., Gindro, K., Viret, O. \& Richter, H. Effects of resveratrol, viniferins and pterostilbene on Plasmopara viticola zoospore mobility and disease development. Vitis 43, 145-148 (2004).

10. Pezet, R., Gindro, K., Viret, O. \& Spring, J. L. Glycosylation and oxidative dimerization of resveratrol are respectively associated to sensitivity and resistance of grapevine cultivars to downy mildew. Physiological Mol. Plant Pathol. 65, 297-303 (2004).

11. Bavaresco, L., \& Fregoni, C. Physiological role and molecular aspects of grapevine stilbenic compounds. In Molecular Biology \& Biotechnology of the Grapevine (ed. Roubelakis-Angelakis, K. A.) 153-182 (Springer, Dordrecht, 2001).

12. Bavaresco, L., Fregoni, C., de Macedo Basto, M. V. Z., \& Vezzulli, S. Physiology \& molecular biology of grapevine stilbenes: an update. In Grapevine Molecular Physiology \& Biotechnology (ed. Roubelakis-Angelakis, K. A.) 341-364 (Springer, Dordrecht, 2009).

13. Dubrovina, A. S. \& Kiselev, K. V. Regulation of stilbene biosynthesis in plants. Planta 246, 597-623 (2017).

14. Dettmer, K., Aronov, P. A. \& Hammock, B. D. Mass spectrometry-based metabolomics. Mass Spectrom. Rev. 26, 51-78 (2007).

15. Hegeman, A. D. Plant metabolomics - meeting the analytical challenges of comprehensive metabolite analysis. Brief. Funct. genomics 9, 139-148 (2010).

16. Allwood, J. W., Ellis, D. I. \& Goodacre, R. Metabolomic technologies and their application to the study of plants and plant-host interactions. Physiologia Plant. 132, 117-135 (2008)
17. Young, N. D. QTL mapping and quantitative disease resistance in plants. Annu. Rev. Phytopathol. 34, 479-501 (1996).

18. Chambers, A. H. et al. Identification of a strawberry flavor gene candidate using an integrated genetic-genomic-analytical chemistry approach. BMC genomics 15, 217 (2014).

19. Eduardo, I. et al. Genetic dissection of aroma volatile compounds from the essential oil of peach fruit: QTL analysis and identification of candidate genes using dense SNP maps. Tree Genet. Genomes 9, 189-204 (2013).

20. Liu, Z. et al. Identification of a Solanum pennellii chromosome 4 fruit flavor and nutritional quality-associated metabolite QTL. Front. plant Sci. 7, 1671 (2016).

21. Tieman, D. M. et al. Identification of loci affecting flavour volatile emissions in tomato fruits. J. Exp. Bot. 57, 887-896 (2006).

22. Toubiana, D. et al. Combined correlation-based network and MQTL analyses efficiently identified loci for branched-chain amino acid, serine to threonine, and proline metabolism in tomato seeds. Plant J. 81, 121-133 (2015).

23. McCallum, S. et al. Genetic and environmental effects influencing fruit colour and QTL analysis in raspberry. Theor. Appl. Genet. 121, 611-627 (2010).

24. Riedelsheimer, $\mathrm{C}$. et al. Genome-wide association mapping of leaf metabolic profiles for dissecting complex traits in maize. Proc. Natil Acad. Sci. USA 109, 8872-8877 (2012)

25. Vosman, B. et al. QTL mapping of insect resistance components of Solanum galapagense. Theor. Appl. Genet. 132, 531-541 (2019).

26. Yang, S. et al. Next generation mapping of enological traits in an F2 interspecific grapevine hybrid family. PLOS ONE 11, e0149560 (2016).

27. Pawlus, A. D. et al. Stilbenoid profiles of canes from Vitis and Muscadinia species. J. Agric. Food Chem. 61, 501-511 (2013).

28. Schnee, S., Viret, O. \& Gindro, K. Role of stilbenes in the resistance of grapevine to powdery mildew. Physiological Mol. Plant Pathol. 72, 128-133 (2008).

29. Vezzulli, S. et al. The Rpv3-3 haplotype and stilbenoid induction mediate downy mildew resistance in a grapevine interspecific population. Front. Plant Sci. 10, 234 (2019).

30. Di Gaspero, G. et al. Selective sweep at the Rpv3 locus during grapevine breeding for downy mildew resistance. Theor. Appl. Genet. 124, 277-286 (2012).

31. Zyprian, E. et al. Quantitative trait loci affecting pathogen resistance and ripening of grapevines. Mol. Genet. Genomics 291, 1573-1594 (2016).

32. Riaz, S., Tenscher, A. C., Ramming, D. W. \& Walker, M. A. Using a limited mapping strategy to identify major QTLs for resistance to grapevine powdery mildew (Erysiphe necator) and their use in marker-assisted breeding. Theor. Appl. Genet. 122, 1059-1073 (2011)

33. Singh, B. \& Sharma, R. A. Plant terpenes: defense responses, phylogenetic analysis, regulation and clinical applications. 3 Biotech 5, 129-151 (2015)

34. Treutter, D. Significance of flavonoids in plant resistance and enhancement of their biosynthesis. Plant Biol. 7, 581-591 (2005).

35. Berger, S., Sinha, A. K. \& Roitsch, T. Plant physiology meets phytopathology: plant primary metabolism and plant-pathogen interactions. J. Exp. Bot. 58, 4019-4026 (2007).

36. Bolton, M. D. Primary metabolism and plant defense-fuel for the fire. Mol. plant-microbe Interact. 22, 487-497 (2009).

37. Schwachtje, J. \& Baldwin, I. T. Why does herbivore attack reconfigure primary metabolism? Plant Physiol. 146, 845-851 (2008).

38. Grimplet, J. et al. Comparative analysis of grapevine whole-genome gene predictions, functional annotation, categorization and integration of the predicted gene sequences. BMC Res. Notes 5, 213 (2012).

39. Fennell, A., Mathiason, K. \& Luby, J. Genetic segregation for indicators of photoperiod control of dormancy induction in Vitis species. Acta Horticulturae 689, 533-540 (2005).

40. Fennell, A., Alahakoon, D., Luby, J. \& Clark, M. Mapping the genetic architecture of grapevine bud growth cycling quantitative traits. XII Int. Conf. Grapevine Breed. Genet. 1248, 337-344 (2018).

41. Smith, C. A., Want, E. J., O'Maille, G., Abagyan, R. \& Siuzdak, G. XCMS: processing mass spectrometry data for metabolite profiling using nonlinear peak alignment, matching, and identification. Anal. Chem. 78, 779-787 (2006).

42. Broman, K. W., Wu, H., Sen, S. \& Churchill, G. A. R/qtl: QTL mapping in experimental crosses. Bioinformatics 19, 889-890 (2003).

43. Grimplet, J. et al. VitisNet: "Omics" integration through grapevine molecular networks. PLoS ONE 4, e8365 (2009).

44. Osier, M. V. VitisPathways: gene pathway analysis for "V. vinifera". Vitis: J. Grapevine Res. 55, 129-133 (2016). 